MATHEMATICS OF COMPUTATION

Volume 68, Number 227, Pages 1057-1066

S 0025-5718(99)01053-4

Article electronically published on February 10, 1999

\title{
EIGENVALUES OF PERIODIC STURM-LIOUVILLE PROBLEMS BY THE SHANNON-WHITTAKER SAMPLING THEOREM
}

\author{
AMIN BOUMENIR
}

\begin{abstract}
We are concerned with the computation of eigenvalues of a periodic Sturm-Liouville problem using interpolation techniques in Paley-Wiener spaces. We shall approximate the Hill discriminant by sampling a few of its values and then find its zeroes which are the square roots of the eigenvalues. Computable error estimates are provided together with eigenvalue enclosures.
\end{abstract}

\section{INTRODUCTION}

We would like to introduce a new method for computing the eigenvalues of classical regular Sturm-Liouville problems with periodic boundary conditions

$$
\left\{\begin{array}{c}
-\varphi^{\prime \prime}(t, \mu)+Q(t) \varphi(t, \mu)=\mu^{2} \varphi(t, \mu) \quad t \in[0, \omega] \\
\varphi(0, \mu)=\varphi(\omega, \mu) \quad \text { and } \varphi^{\prime}(0, \mu)=\varphi^{\prime}(\omega, \mu),
\end{array}\right.
$$

where $Q(t) \in L^{1}(0, \omega)$. For the spectral theory of periodic differential equations, we shall refer to [4], [1] and [8]. Recall that in general the spectrum of (1.1) may not be simple as in the case of separated boundary conditions, and this is a major difficulty.

In this work, we shall extend the method in [3], which relies on the interpolation in Paley-Wiener spaces of a certain boundary function, whose zeros are square roots of eigenvalues. In our case the boundary function turns out to be the wellknown Hill discriminant (see [4]) and only few values are needed for its approximation. The truncation error can be minimized by increasing the number of sampling points, which gives higher accuracy on the numerical side and provides eigenvalue enclosures. We shall examine a few examples, where eigenvalue enclosures and comparisons of the results with the well-known code Sleign2 are provided. In the last example our interpolation scheme is compared with an "exact" solution. One of the features of the method is the possibility of locating double eigenvalues. Indeed, by minimizing the truncation error, we can zoom in on close eigenvalues, and if they happen to be simple, then we can find two disjoint enclosures separating them.

\section{HILL'S DISCRIMINANT}

The Hill discriminant, which is at the heart of the spectral theory of the periodic Sturm-Liouville operator, is defined by

$$
\Delta(\mu):=\varphi_{1}(\omega, \mu)+\varphi_{2}^{\prime}(\omega, \mu)
$$

Received by the editor June 9, 1997 and, in revised form, October 28, 1997.

1991 Mathematics Subject Classification. Primary 34L15, 42 A15.

Key words and phrases. Periodic Sturm-Liouville problems, eigenvalue problem, interpolation, Shannon-Whittaker sampling theorem. 
where $\varphi_{1}(t, \mu)$ and $\varphi_{2}(t, \mu)$ are two independent solutions of (1.1) satisfying

$$
\left\{\begin{array} { l } 
{ \varphi _ { 1 } ( 0 , \mu ) = 1 } \\
{ \varphi _ { 1 } ^ { \prime } ( 0 , \mu ) = 0 }
\end{array} \quad \text { and } \quad \left\{\begin{array}{l}
\varphi_{2}(0, \mu)=0 \\
\varphi_{2}^{\prime}(0, \mu)=1 .
\end{array}\right.\right.
$$

Recall that $\mu^{2}$ is an eigenvalue associated with (1.1) if and only if $\mu$ is solution of

$$
\Delta(\mu)=2
$$

(see [4]). In order to interpolate the Hill discriminant we shall need to find a suitable representation in terms of analytic functions.

From the inverse spectral theory (see [7]) $\varphi_{1}(t, \mu)$ and $\varphi_{2}(t, \mu)$ can be represented by

$$
\begin{aligned}
\varphi_{1}(t, \mu) & =\cos t \mu+\int_{0}^{t} K_{1}(t, \eta) \cos \eta \mu d \eta, \\
\varphi_{2}(t, \mu) & =\frac{\sin t \mu}{\mu}+\int_{0}^{t} K_{2}(t, \eta) \frac{\sin \eta \mu}{\mu} d \eta,
\end{aligned}
$$

where $K_{1}$ and $K_{2}$ are the kernels of transformation operators. We recall that $K_{1}$ and $K_{2}$ have $m+1$ locally integrable derivatives if $Q$ has $m$ locally integrable derivatives. In our case $m=0$, which means that $\frac{\partial}{\partial t} K_{2}$ and $\frac{\partial}{\partial t} K_{1}$ are locally integrable and $K_{1}$ and $K_{2}$ are continuous functions. Thus the integrals in (2.3) are well defined. The Lyapunov function or Hill's discriminant for (1.1) can now be described using $K_{1}$ and $K_{2}$ :

$$
\begin{aligned}
\Delta(\mu)=\varphi_{1}(\omega, \mu) & +\varphi_{2}^{\prime}(\omega, \mu) \\
=2 \cos \omega \mu & +K_{1}(\omega, \omega) \frac{\sin \omega \mu}{\mu}+K_{2}(\omega, \omega) \frac{\sin \omega \mu}{\mu} \\
& +\int_{0}^{\omega}\left[\frac{\partial}{\partial t} K_{2}(\omega, \eta)+\frac{\partial}{\partial t} K_{1}(\omega, \eta)\right] \frac{\sin \eta \mu}{\mu} d \eta .
\end{aligned}
$$

Let us agree to denote the last integral term by

$$
S(\mu):=\int_{0}^{\omega}\left[\frac{\partial}{\partial t} K_{2}(\omega, \eta)+\frac{\partial}{\partial t} K_{1}(\omega, \eta)\right] \frac{\sin \eta \mu}{\mu} d \eta,
$$

and several useful constants by

$$
\begin{gathered}
\left|\frac{\sin z}{z}\right| \leq c \frac{\exp (|\operatorname{Im} z|)}{1+|z|}, \\
q:=\int_{0}^{\omega}|Q(\eta)| d \eta
\end{gathered}
$$

and

$$
\xi:=\exp \left(c \int_{0}^{\omega} \eta|Q(\eta)| d \eta\right) .
$$

We now recall that Paley-Wiener spaces are defined by

$$
P W_{\omega}:=\left\{F(\mu) \text { entire: }|F(\mu)|<M e^{\omega|\operatorname{Im} \mu|} \text { and } \int_{-\infty}^{\infty}|F(\mu)|^{2} d \mu<\infty\right\} .
$$

Also (see [7])

$$
K_{1}(\omega, \omega)=K_{2}(\omega, \omega)=\frac{1}{2} \int_{0}^{\omega} Q(\eta) d \eta .
$$


Thus in order to recover

$$
\Delta(\mu):=2 \cos (\omega \mu)+\frac{\sin \omega \mu}{\mu} \int_{0}^{\omega} Q(\eta) d \eta+S(\mu)
$$

we need to find $S(\mu)$ only.

Proposition 2.1. If $Q(t) \in L^{1}(0, \omega)$, then

$$
S(\mu) \in P W_{\omega} \quad \text { and } \quad E_{1}:=\sqrt{\int_{-\infty}^{\infty}|\mu S(\mu)|^{2} d \mu} \leq \xi c^{2} q^{2} \sqrt{\frac{3}{2} \omega}
$$

Proof. Because $Q(t)$ is locally integrable, it follows that $\left[\frac{\partial}{\partial t} K_{2}(\omega, \eta)+\frac{\partial}{\partial t} K_{1}(\omega, \eta)\right]$ is also locally integrable, and applying the Riemann-Lebesgue theorem to (2.5) we deduce that $S(\mu)=o\left(\frac{1}{\mu}\right)$, i.e., $S(\mu) \in L_{d \mu}^{2}(-\infty, \infty)$. Next observe that

$$
\begin{aligned}
|S(\mu)| & \leq \int_{0}^{\omega}\left|\frac{\partial}{\partial t} K_{2}(\omega, \eta)+\frac{\partial}{\partial t} K_{1}(\omega, \eta)\right| \frac{c \eta \exp (\eta|\operatorname{Im} \mu|)}{1+|\eta \mu|} d \eta \\
& \leq \int_{0}^{\omega}\left|\frac{\partial}{\partial t} K_{2}(\omega, \eta)+\frac{\partial}{\partial t} K_{1}(\omega, \eta)\right| \frac{c \eta}{1+|\eta \mu|} d \eta \exp (\omega|\operatorname{Im} \mu|) \\
& \leq C_{1} \exp (\omega|\operatorname{Im} \mu|) .
\end{aligned}
$$

Hence $S(\mu) \in P W_{\omega}$. If $C_{1}$ is known, then we can estimate the constant $E_{1}$, which is crucial in the computation of error bounds. To this end we shall use the following integral equations (see [10]):

$$
\begin{aligned}
\varphi_{1}(t, \mu) & =\cos (t \mu)+T_{\mu} \varphi_{1}(t, \mu), \\
\varphi_{2}(t, \mu) & =\frac{\sin (t \mu)}{\mu}+T_{\mu} \varphi_{2}(t, \mu),
\end{aligned}
$$

where

$$
\begin{gathered}
C(0, \omega) \stackrel{T_{\mu}}{\longrightarrow} C(0, \omega), \\
T_{\mu} f(t):=\int_{0}^{t} \frac{\sin (\mu(t-\eta))}{\mu} Q(\eta) f(\eta) d \eta, \quad t \in(0, \omega), \\
\left\|T_{\mu}\right\| \leq \int_{0}^{\omega} \frac{c(\omega-\eta)}{1+|\mu|(\omega-\eta)}|Q(\eta)| d \eta \\
\leq c q \frac{\omega}{1+|\mu| \omega} .
\end{gathered}
$$

For $\mu$ large enough, the method of successive approximation yields (see [10])

$$
\varphi_{1}(t, \mu)=\sum_{n \geq 0} T_{\mu}^{n} \cos (t \mu) \quad \text { and } \quad \varphi_{2}(t, \mu)=\sum_{n \geq 0} T_{\mu}^{n} \frac{\sin \eta \mu}{\mu},
$$

and by the Gronwall lemma applied to (2.7) we obtain

$$
\begin{aligned}
\left|\varphi_{1}(t, \mu)\right| \leq \exp \left(\int_{0}^{t} \frac{c \eta|Q(\eta)|}{1+|\mu| \eta} d \eta\right) \leq \exp \left(c \int_{0}^{t} \eta|Q(\eta)| d \eta\right) \\
\left|\varphi_{2}(t, \mu)\right| \leq c \frac{t}{1+|\mu| t} \exp \left(\int_{0}^{t} \frac{c \eta|Q(\eta)|}{1+|\mu| \eta} d \eta\right) \\
\leq c \xi \frac{t}{1+|\mu| t} .
\end{aligned}
$$


Applying $T_{\mu}$ to (2.7) yields

$$
\varphi_{1}(t, \mu)=\cos (t \mu)+T_{\mu} \cos (t \mu)+T_{\mu}^{2} \varphi_{1}(t, \mu),
$$

where

$$
\begin{aligned}
\left|T_{\mu}^{2} \varphi_{1}(\omega, \mu)\right| & \leq \frac{(c q \omega)^{2}}{(1+|\mu| \omega)^{2}} \exp \int_{0}^{t} \frac{c \eta|Q(\eta)|}{1+|\mu| \eta} d \eta \\
& \leq \frac{(c q \omega)^{2}}{(1+|\mu| \omega)^{2}} \exp \left(c \int_{0}^{t} \eta|Q(\eta)| d \eta\right) \\
& \leq \xi(c q \omega)^{2} \frac{1}{(1+|\mu| \omega)^{2}} .
\end{aligned}
$$

Similarly we have

$$
\begin{aligned}
\varphi_{2}(t, \mu) & =\frac{\sin (t \mu)}{\mu}+T_{\mu} \frac{\sin (t \mu)}{\mu}+T_{\mu}^{2} \varphi_{2}(t, \mu), \\
\varphi_{2}^{\prime}(t, \mu) & =\cos (t \mu)+\frac{d}{d t} T_{\mu} \frac{\sin (t \mu)}{\mu}+\frac{d}{d t} T_{\mu}^{2} \varphi_{2}(t, \mu),
\end{aligned}
$$

where

$$
\begin{aligned}
\left|\frac{d}{d t} T_{\mu}^{2} \varphi_{2}(\omega, \mu)\right| & =\left|\int_{0}^{\omega} \cos (\mu(\omega-t)) Q(t) \int_{0}^{t} \frac{\sin (\mu(t-\eta))}{\mu} Q(\eta) \varphi_{2}(\eta, \mu) d \eta d t\right| \\
& \leq \int_{0}^{\omega}|Q(t)| \int_{0}^{t} \frac{c(t-\eta)}{1+|\mu|(t-\eta)}|Q(\eta)|\left|\varphi_{2}(\eta, \mu)\right| d \eta d t \\
& \leq \int_{0}^{\omega}|Q(t)| \int_{0}^{t} \frac{c \eta}{1+|\mu| \eta}|Q(\eta)| \xi c \frac{\eta}{1+|\mu| \eta} d \eta d t \\
& \leq \xi c^{2} \int_{0}^{\omega}|Q(t)| \int_{0}^{t}\left(\frac{\eta}{1+|\mu| \eta}\right)^{2}|Q(\eta)| d \eta d t \\
& \leq \xi c^{2}\left(\frac{\omega}{1+|\mu| \omega}\right)^{2} \int_{0}^{\omega}|Q(t)| \int_{0}^{t}|Q(\eta)| d \eta d t \\
& \leq \frac{\xi c^{2}}{2}\left(\frac{\omega}{1+|\mu| \omega}\right)^{2}\left(\int_{0}^{\omega}|Q(t)| d t\right)^{2} \\
& \leq \frac{1}{2} \xi c^{2} q^{2} \omega^{2} \frac{1}{(1+|\mu| \omega)^{2}} .
\end{aligned}
$$

We now use (2.9) and (2.11) to obtain

$$
\begin{aligned}
\Delta(\mu) & =\varphi_{1}(\omega, \mu)+\varphi_{2}^{\prime}(\omega, \mu) \\
& =2 \cos (t \mu)+T_{\mu} \cos (t \mu)+\frac{d}{d t} T_{\mu} \frac{\sin (t \mu)}{\mu}+T_{\mu}^{2} \varphi_{1}(t, \mu)+\frac{d}{d t} T_{\mu}^{2} \varphi_{2}(t, \mu) .
\end{aligned}
$$

Observe that

$$
\begin{aligned}
T_{\mu} \cos (t \mu)+\frac{d}{d t} T_{\mu} \frac{\sin (t \mu)}{\mu} \\
=\int_{0}^{t}\left(\frac{\sin (\mu(t-\eta))}{\mu} \cos (\eta \mu)+\cos (\mu(t-\eta)) \frac{\sin (\eta \mu)}{\mu}\right) Q(\eta) d \eta \\
=\frac{\sin (\mu t)}{\mu} \int_{0}^{t} Q(\eta) d \eta .
\end{aligned}
$$


Thus a new representation of the Hill discriminant follows:

$$
\Delta(\mu)=2 \cos (t \mu)+\frac{\sin (\mu t)}{\mu} \int_{0}^{t} Q(\eta) d \eta+T_{\mu}^{2} \varphi_{1}(t, \mu)+\frac{d}{d t} T_{\mu}^{2} \varphi_{2}(t, \mu) .
$$

Therefore (2.6) together with (2.13) imply

$$
S(\mu)=T_{\mu}^{2} \varphi_{1}(t, \mu)+\frac{d}{d t} T_{\mu}^{2}\left(\varphi_{2}(t, \mu)\right)
$$

and estimates (2.12) and (2.10) yield

$$
|S(\mu)| \leq \frac{3 \xi c^{2} q^{2} \omega^{2}}{2} \frac{1}{(1+|\mu| \omega)^{2}} .
$$

Hence it follows from (2.14) and (2.15) that $S(\mu) \in P W_{\omega}$ and

$$
E_{1}=\sqrt{\int|\mu S(\mu)|^{2} d \mu} \leq \frac{3 \xi c^{2}}{2} q^{2} \omega^{2} \sqrt{\frac{2}{3 \omega^{3}}} \leq \xi c^{2} q^{2} \sqrt{\frac{3}{2} \omega} .
$$

It is also possible to use asymptotics of solutions (see [5]) to derive the estimates above.

\section{General Case}

We now address the question of how to apply the idea above to more general equations, such as

$$
\left\{\begin{array}{l}
-\left(p(x) y^{\prime}(x, \mu)\right)^{\prime}+q(x) y(x, \mu)=\mu^{2} s(x) y(x, \mu), \quad 0<x<a \\
y(0, \mu)=y(a, \mu), \quad p(0) y^{\prime}(0, \mu)=p(a) y^{\prime}(a, \mu),
\end{array}\right.
$$

where $p(x), q(x)$, and $s(x)$ are real valued, $\frac{1}{p(x)}, q(x), s(x) \in L^{1}(0, a)$ and $s(x) \geq$ 0 . It is well known that $\mu_{n}^{2}$ (see [4]) is an eigenvalue of (3.1) if and only if $\mu_{n}$ is a root of

$$
D(\mu):=\psi_{1}(a, \mu)+p(a) \psi_{2}^{\prime}(a, \mu)=2,
$$

where $\psi_{1}(x, \mu)$ and $\psi_{2}(x, \mu)$ are two independent solutions of

$$
\left(p(x) y^{\prime}(x, \mu)\right)^{\prime}+q(x) y(x, \mu)=\mu^{2} s(x) y(x, \mu), \quad 0<x<a,
$$

with initial conditions

$$
\left\{\begin{array} { l } 
{ \psi _ { 1 } ( 0 , \mu ) = 1 } \\
{ p ( 0 ) \psi _ { 1 } ^ { \prime } ( 0 , \mu ) = 0 }
\end{array} \quad \text { and } \quad \left\{\begin{array}{l}
\psi_{2}(0, \mu)=0 \\
p(0) \psi_{2}^{\prime}(0, \mu)=1 .
\end{array}\right.\right.
$$

It is readily seen that $D(\mu)$ is an entire function of $\mu$ of order one, and the main difficulty is estimating its type. Once the type is obtained, which depends on $p(x), q(x)$ and $s(x)$, we can apply the above method with a few modifications.

One simple way to deal with (3.1) is to transform it to the standard form (1.1) which is possible when the coefficients $\frac{1}{p(x)}$ and $s(x)$ are $C^{2}(0, a)$, and $s(x) \geq \delta>0$. Indeed, the Liouville-Green transformation allows us to recast equation (3.1) into an equivalent periodic problem (see [4])

$$
\left\{\begin{array}{lr}
-\varphi^{\prime \prime}(t, \mu)+Q(t) \varphi(t, \mu)=\mu^{2} \varphi(t, \mu) \quad t \in[0, A] \\
\varphi(0, \mu)=\varphi(A, \mu) \quad \text { and } \quad \varphi^{\prime}(0, \mu)=K \varphi^{\prime}(A, \mu)
\end{array}\right.
$$


where $t(x)=\int_{0}^{x} \sqrt{\frac{s(\eta)}{p(\eta)}} d \eta$ for $x \in[0, a]$ and

$$
\varphi(t(x), \mu)=y(x, \mu), Q(t(x))=q(x)-p^{\frac{1}{4}}(x) s^{-\frac{3}{4}}(x) \frac{d}{d x} p(x) \frac{d}{d x}\{p(x) s(x)\}^{\frac{-1}{4}} .
$$

Clearly the type of $D(\mu)$ is then $A:=t(a)$ and the boundary conditions are obtained from $y^{\prime}(x, \mu):=\varphi^{\prime}(t(x), \mu) \sqrt{\frac{s(x)}{p(x)}}$, and so $K:=\sqrt{\frac{s(\omega) p(\omega)}{s(0) p(0)}}$. In case $s(\omega) p(\omega)=s(0) p(0)$, then we have $K=1$.

- If $p(x)$ and $s(x)$ are not smooth, then we can still use ideas from the transformation operators for the string (see [2])

$$
-p(x)\left(p(x) y^{\prime}(x, \mu)\right)^{\prime}+p(x) q(x) y(x, \mu)=\mu^{2} p(x) s(x) y(x, \mu) \quad 0<x<a .
$$

Then setting $t(x):=\int_{0}^{x} \frac{1}{p(\eta)} d \eta, \gamma:=t(a)$ and $\varphi(t(x))=y(x)$ we have

$$
\begin{gathered}
-\varphi^{\prime \prime}(t, \mu)+Q(t) \varphi(t, \mu)=\mu^{2} \omega(t) \varphi(t, \mu) \quad 0<t<\gamma \\
Q(t):=p(x(t)) q(x(t)) \quad \text { and } \quad \omega(t):=p(x(t)) s(x(t)) .
\end{gathered}
$$

If the spectrum is bounded from below, then a simple transformation (see [6], p. 90) allows us to recast (3.4) into

$$
-\psi^{\prime \prime}(t, \mu)=\mu^{2} W(t) \psi(t, \mu)
$$

and an upper bound for the type of solutions $\psi(t, \mu)$ is given by $\sqrt{2 \gamma \int_{0}^{\gamma} W(t) d t}$ (see [6], p. 32).

- There are other methods which can be used to estimate the type, such as semi-classical approximation. The asymptotic distribution of eigenvalues of (3.5) depends on the behaviour of the weight $W(t)$ as $t \rightarrow 0$.

\section{SAMPLING}

Proposition 2.1 allows us to use the well-known Whittaker-Shannon-Kotelnikov sampling theorem to reconstruct the function $S(\mu)$ from its sampling values (see [9] and [11])

$$
S(\mu):=\sum_{k=-\infty}^{\infty} S\left(\frac{k \pi}{\omega}\right) \frac{\sin (\omega \mu-k \pi)}{\omega \mu-k \pi},
$$

where $S\left(\frac{k \pi}{\omega}\right)$ are obtained by using (2.14) where the values of

$$
\Delta\left(\frac{k \pi}{\omega}\right)=\varphi_{1}\left(\omega, \frac{k \pi}{\omega}\right)+\varphi_{2}^{\prime}\left(\omega, \frac{k \pi}{\omega}\right)
$$

are computed by solving the initial value problem defined by (1.1) and (2.1). A good approximation of $S(\mu)$ can then be obtained using very few points

$$
S_{N}(\mu):=\sum_{k=-N}^{N} S\left(\frac{k \pi}{\omega}\right) \frac{\sin (\omega \mu-k \pi)}{\omega \mu-k \pi}
$$

and the truncation error

$$
\left|S(\mu)-S_{N}(\mu)\right| \leq T_{N}(\mu)
$$

can be easily estimated for $|\mu|<\frac{N \pi}{\omega}$ (see [11]),

$$
T_{N}(\mu):=\frac{E_{1}}{N+1} \frac{2 \omega \sqrt{\omega}|\sin \omega \mu|}{\pi^{2} \sqrt{3}}\left(\frac{1}{\sqrt{N \pi-\omega \mu}}+\frac{1}{\sqrt{N \pi+\omega \mu}}\right),
$$


where, by Proposition 2.1, $E_{1} \leq \xi c^{2} q^{2} \sqrt{\frac{3}{2} \omega}$. It is essential to have an upper bound estimate on the magnitude of constant $E_{1}$ in terms of $Q(t)$ in order to obtain precise error bounds on the eigenvalues and possible enclosures.

The knowledge of $S(\mu)$ allows us to reconstruct the Hill discriminant $\Delta(\mu)$ defined by (2.6) and then solve (2.2), which we shall agree to write as

$$
\widetilde{\Delta}(\mu):=\Delta(\mu)-2=0
$$

to find the eigenvalues for $|\mu|<\frac{N \pi}{\omega}$. It is readily seen that $S_{N}(\mu)$ helps define an approximation to $\widetilde{\Delta}$,

$$
\widetilde{\Delta}_{N}(\mu):=2 \cos (\omega \mu)+\frac{\sin (\mu \omega)}{\mu} \int_{0}^{\omega} Q(\eta) d \eta+S_{N}(\mu)-2 .
$$

Let $\mu_{N}$ be a solution of $\widetilde{\Delta}_{N}\left(\mu_{N}\right)=0$ and denote by $\mu^{*}$ a solution $\widetilde{\Delta}\left(\mu^{*}\right)=0$. In other words $\left(\mu^{*}\right)^{2}$ is an eigenvalue and $\left(\mu_{N}\right)^{2}$ is its approximation. Since

$$
\left|\widetilde{\Delta}_{N}(\mu)-\widetilde{\Delta}(\mu)\right| \leq T_{N}(\mu)
$$

we have

$$
\left|\widetilde{\Delta}_{N}\left(\mu^{*}\right)\right| \leq T_{N}\left(\mu^{*}\right)
$$

and thus the enclosure is defined by

$$
\mu^{*} \in I_{\mu_{N}}:=\left\{\mu \text { such that }\left|\widetilde{\Delta}_{N}(\mu)\right| \leq T_{N}(\mu)\right\} .
$$

Observe that the endpoints of $I_{\mu_{N}}$ are solutions of $\widetilde{\Delta}_{N}(\mu)= \pm T_{N}(\mu)$, and so they are computable.

From the uniform convergence of $\widetilde{\Delta}_{N}(\mu) \rightarrow \widetilde{\Delta}(\mu)$, we distinguish two cases.

- $\widetilde{\Delta}^{\prime}\left(\mu^{*}\right) \neq 0$, i.e. $\mu^{*}$ is a simple eigenvalue and so there exists an $N_{0}$,

$$
\begin{array}{ll} 
& \inf _{\mu \in I_{\mu_{N_{0}}}}\left|\widetilde{\Delta}_{N_{0}}^{\prime}(\mu)\right|=\delta\left(N_{0}\right)>0 \\
\text { and } \forall N \geq N_{0} \quad & \inf _{\mu \in I_{\mu_{N}}}\left|\widetilde{\Delta}_{N}^{\prime}(\mu)\right|>0 .
\end{array}
$$

Proposition 4.1. Let $\mu^{*}>0$, be a simple eigenvalue of (1.1), then $\forall \varepsilon>0$, $\exists N_{0}$ such that $\forall N \geq N_{0}, \exists \mu_{N}$ such that

$$
\left|\mu^{*}-\mu_{N}\right|<\frac{\varepsilon}{\inf _{\mu \in I_{\mu_{N}}}\left|\widetilde{\Delta}_{N}^{\prime}(\mu)\right|} .
$$

Proof. Given $\mu^{*}$, we can find $N$, such that $\mu^{*}<\frac{N \pi}{\omega}, \inf _{\mu \in I_{\mu_{N}}}\left|\widetilde{\Delta}_{N}^{\prime}(\mu)\right| \geq \delta>0$ and $T_{N}(\mu)<\varepsilon$ for $\mu \in I_{\mu_{N}}$. From $\left|\widetilde{\Delta}_{N}\left(\mu^{*}\right)-\widetilde{\Delta}_{N}\left(\mu_{N}\right)\right| \leq T_{N}\left(\mu^{*}\right)$, the mean value theorem implies

$$
\left|\left(\mu^{*}-\mu_{N}\right) \widetilde{\Delta}_{N}^{\prime}(\eta)\right| \leq T_{N}\left(\mu^{*}\right), \quad \eta \in\left(\mu^{*}, \mu_{N}\right) \subset I_{\mu_{N}},
$$

and since $\mu^{*}$ is simple, $\inf _{\mu \in I_{\mu_{N}}}\left|\widetilde{\Delta}_{N}^{\prime}(\mu)\right|>0$, and we have

$$
\left|\mu^{*}-\mu_{N}\right| \leq \frac{T_{N}\left(\mu^{*}\right)}{\inf _{\mu \in I_{\mu_{N}}}\left|\widetilde{\Delta}_{N}^{\prime}(\mu)\right|} \leq \frac{\varepsilon}{\inf _{\mu \in I_{\mu_{N}}}\left|\widetilde{\Delta}_{N}^{\prime}(\mu)\right| .}
$$


As we increase $N \geq N_{0}, \sup _{\mu \in I_{\mu_{N}}} T_{N}(\mu) \rightarrow 0, \inf _{\mu \in I_{\mu_{N}}}\left|\widetilde{\Delta}_{N}^{\prime}(\mu)\right| \rightarrow \delta\left(N_{0}\right)$, and so $\left|\mu^{*}-\mu_{N}\right| \rightarrow 0$ as $N \rightarrow \infty$.

- The second case is when we have a double eigenvalue, which is characterized by

$$
\widetilde{\Delta}\left(\mu^{*}\right)=0, \widetilde{\Delta}^{\prime}\left(\mu^{*}\right)=0 \text { and } \widetilde{\Delta}^{\prime \prime}\left(\mu^{*}\right) \neq 0 .
$$

Because of the concavity of the $\widetilde{\Delta}(\mu)$ at $\mu^{*}$, there are two cases.

- $\widetilde{\Delta}_{N}(\mu)$ is also concave and may not have zeroes. In this case we look for solutions inside the intervals defined by (4.2).

- If $\widetilde{\Delta}_{N}\left(\mu_{N}\right)$ has two zeroes then

$$
\begin{aligned}
& \widetilde{\Delta}_{N}\left(\mu^{*}\right)=\widetilde{\Delta}_{N}\left(\mu_{N}\right)+\left(\mu^{*}-\mu_{N}\right) \widetilde{\Delta}_{N}^{\prime}\left(\mu_{N}\right)+\frac{1}{2}\left(\mu^{*}-\mu_{N}\right)^{2} \widetilde{\Delta}_{N}^{\prime \prime}(\eta), \\
& \widetilde{\Delta}_{N}\left(\mu^{*}\right)=\widetilde{\Delta}\left(\mu^{*}\right)+\left(\mu^{*}-\mu_{N}\right) \widetilde{\Delta}_{N}^{\prime}\left(\mu_{N}\right)+\frac{1}{2}\left(\mu^{*}-\mu_{N}\right)^{2} \widetilde{\Delta}_{N}^{\prime \prime}(\eta), \\
& \widetilde{\Delta}_{N}\left(\mu^{*}\right)-\widetilde{\Delta}\left(\mu^{*}\right)=\left(\mu^{*}-\mu_{N}\right) \widetilde{\Delta}_{N}^{\prime}\left(\mu_{N}\right)+\frac{1}{2}\left(\mu^{*}-\mu_{N}\right)^{2} \widetilde{\Delta}_{N}^{\prime \prime}(\eta), \\
& \frac{1}{2}\left(\mu^{*}-\mu_{N}\right)^{2} \widetilde{\Delta}_{N}^{\prime \prime}(\eta)+\left(\mu^{*}-\mu_{N}\right) \widetilde{\Delta}_{N}^{\prime}\left(\mu_{N}\right)-\left[\widetilde{\Delta}_{N}\left(\mu^{*}\right)-\widetilde{\Delta}\left(\mu^{*}\right)\right]=0,
\end{aligned}
$$

where $\left[\widetilde{\Delta}_{N}\left(\mu^{*}\right)-\widetilde{\Delta}\left(\mu^{*}\right)\right] \leq T_{N}\left(\mu^{*}\right)$. Clearly the coefficients of this quadratic are intervals, i.e., $\inf _{\eta \in I_{\mu}} \widetilde{\Delta}_{N}^{\prime \prime}(\eta) \leq \widetilde{\Delta}_{N}^{\prime \prime}(\eta) \leq \max _{\eta \in I_{\mu}} \widetilde{\Delta}_{N}^{\prime \prime}(\eta)$, and similarly $0 \leq T_{N}\left(\mu^{*}\right) \leq \max _{\eta \in I_{\mu}} T_{N}(\eta)$. Therefore we can find an upper bound for $\left|\mu^{*}-\mu_{N}\right|$, using interval analysis. Since $\widetilde{\Delta}_{N}\left(\mu_{N}\right)$ may have no roots in the neighbourhood of $\mu^{*}$, the only practical way to locate double eigenvalues is the interval defined by (4.2). In practice it is more convenient to be given $N$ and then compute the truncation error $T_{N}(\mu)$ to find the intervals $I_{\mu_{N}}$ defined by (4.2).

\section{EXAMPLES}

We shall consider problems defined by (1.1), where $Q(x)$ is integrable. Recall that $N$ represents the number of points used in the Shannon-Whittaker sampling theorem. The positive eigenvalues are obtained by recovering the Hill discriminant from its sampled values. To this end we only need to compute the values $\varphi_{1}(\omega, \mu)+$ $\varphi_{2}^{\prime}(\omega, \mu)$ for $\mu=k \frac{\pi}{\omega}, k=0,1, \ldots, N$, which are obtained by solving the initial value problem (1.1) with Runge-Kutta 4-5, which is implemented with the symbolic manipulator Maple with precision set to 13 digits.

- Example 1: Let us choose $\omega=2$, and

$$
q(x):=\left\{\begin{array}{cl}
0 & 0<x<1 / 2 \\
\frac{1}{1+x^{2}} & 1 / 2<x<7 / 4 \\
0 & 7 / 4<x<2
\end{array}\right.
$$

The square roots of the eigenvalues are listed below.

\begin{tabular}{|l|l|l|l|l|l|}
\hline $\mathrm{N}$ & $\mu_{1}$ & $\mu_{2}$ & $\mu_{3}$ & $\mu_{4}$ & $\mu_{5}$ \\
\hline 5 & 0.5376360812 & 3.141592653 & 3.198533821 & 6.283185307 & 6.309309048 \\
\hline 13 & 0.5376389426 & 3.141592653 & 3.198655062 & 6.283185307 & 6.309851843 \\
\hline 19 & 0.5376389987 & 3.141592654 & 3.198665145 & 6.283185307 & 6.309892873 \\
\hline
\end{tabular}


The enclosure for the first simple eigenvalue $\mu_{1}^{2}$ is given below.

\begin{tabular}{|l|l|l|l|}
\hline $\mathrm{N}$ & $\mu_{\min }$ & $\mu_{1}$ & $\mu_{\max }$ \\
\hline 5 & 0.5063 & 0.5376360812 & 0.5700 \\
\hline 13 & 0.5292 & 0.5376389426 & 0.5461 \\
\hline 19 & 0.5327 & 0.5376389987 & 0.5425 \\
\hline
\end{tabular}

- Example 2: $q(x):=2 x(1-x)$ and $\omega=1$.

The enclosures are given below.

\begin{tabular}{|l|l|l|l|}
\hline $\mathrm{N}$ & $\mu_{1 \mathrm{~min}}$ & $\mu_{1}$ & $\mu_{1 \max }$ \\
\hline 4 & 0.576496 & 0.576890580 & 0.577287 \\
\hline 7 & 0.575138 & 0.576891292 & 0.578648 \\
\hline 15 & 0.576292 & 0.576891400 & 0.577491 \\
\hline 20 & 0.576496 & 0.576891408 & 0.577287 \\
\hline
\end{tabular}

\begin{tabular}{|l|l|l|l|l|}
\hline $\mathrm{N}$ & $\mu_{2,3 \min }$ & $\mu_{2}$ & $\mu_{3}$ & $\mu_{2,3 \max }$ \\
\hline 4 & 6.29811294928 & 6.30777169387 & 6.31155085736 & 6.32991780134 \\
\hline 7 & 6.30116798050 & 6.30769833630 & 6.31163581017 & 6.32196796225 \\
\hline 15 & 6.30414770889 & 6.30768224351 & 6.31165451250 & 6.31645456422 \\
\hline 20 & 6.30496865578 & 6.30768111508 & 6.31165582479 & 6.31520080034 \\
\hline
\end{tabular}

For $N=20$, an estimate of the truncation error is given by

$$
\begin{gathered}
T N(\mu)<27.10^{-6} \text { when } \mu \in(6.304968,6.3152008) \\
T N(\mu)<46.10^{-5} \quad \mu_{1} \in(0.5764,0.5774) .
\end{gathered}
$$

1. A quick run of Sleign2, with a tolerance of $10^{-7}$, gives the following eigenvalues

$$
\begin{array}{|l|l|l|l|}
\text { Sleign2 } \mu=\sqrt{\lambda} & 0.576919405 & 6.31198463 & 6.31204087 \\
\hline
\end{array}
$$

which agrees with our results. Observe that all three square roots are within our enclosures.

- Example 3: In this example $\omega=3$ and

$$
q(x):=\left\{\begin{array}{cc}
0 & 0<x<1 \\
\frac{\pi}{2} & 1<x<2 \\
0 & 2<x<3
\end{array}\right.
$$

It is a simple task to compute the solutions $\varphi_{1}(t, \mu)$ and $\varphi_{2}(t, \mu)$ explicitly, and so it follows that $\widetilde{\Delta}(\mu)$ is also obtained in a closed form. From the "exact" solution the first five eigenvalues are simple. The interpolation gives satisfactory results as can be seen from the following table.

\begin{tabular}{|l|l|l|l|l|l|}
\hline $\mathrm{N}$ & $\mu_{1}$ & $\mu_{2}$ & $\mu_{3}$ & $\mu_{4}$ & $\mu_{5}$ \\
\hline 6 & 0.6629613387 & 2.162705929 & 2.277446491 & 4.241533696 & 4.261003349 \\
\hline 11 & 0.6629773389 & 2.162572186 & 2.277814772 & 4.240002625 & 4.263173280 \\
\hline 21 & 0.6629803405 & 2.162548286 & 2.277880363 & 4.239796746 & 4.263474201 \\
\hline Exact & 0.6629808831 & 2.162544034 & 2.277892016 & 4.239762398 & 4.263524610 \\
\hline
\end{tabular}




\section{Conclusion}

With a simple analysis, and with values of solutions of initial value problems computed at a few values of the eigenparameter, we have computed the eigenvalues of a periodic problem with a certain estimated error. The main advantage is the recovery of an approximation of the Hill discriminant whose graph shows possible double eigenvalues. By increasing the number of sampling points, the truncation error is reduced and this allows us to zoom in on very close eigenvalues. Observe that the eigenvalues in Example 2, found by the code Sleign2, are within our enclosures.

One of the main features of our scheme is the estimate on the truncation error, $T_{N}(\mu)$ and its use for the eigenvalues enclosures. It is also possible for $T_{N}(\mu)$ to include the phase and amplitude errors made on the sampling values.

\section{ACKNOWLEDGMENT}

The author acknowledges the support of K.F.U.P.M.

\section{REFERENCES}

[1] P. B. Bailey, W. N. Everitt and A. Zettl, Regular and singular Sturm-Liouville problem, with coupled boundary conditions, Proc. Roy. Soc. Edinburgh Sect. A, 126, 1995, 505-514. MR 97c:34037

[2] A. Boumenir, Transmutation operator for $\frac{1}{w(x)} D^{2}$, Proc. Roy. Soc. Edinburgh Sect. A. 125, 1995, 85-98. MR 95m:34047

[3] A. Boumenir and B. Chanane, Eigenvalues of S-L Systems using Sampling Theory, Applicable Analysis, Vol. 62, 1996, 323-334.

[4] M. S. P. Eastham, The spectral theory of periodic differential equations, Scottish Academic Press, 1973.

[5] C. T. Fulton and S. A. Pruess, Eigenvalues and eigenfunctions asymptotics for regular SturmLiouville problems, J. Math. Anal. Appl, 188, 1994, 297-340. MR 97f:34071

[6] I. S. Kac and M. G. Krein, Spectral function of the string, Amer. Math. Soc. Transl. (2), 103, 1970, 19-103.

[7] B. M. Levitan, Inverse Sturm-Liouville Problems, VNU Science Press, The Netherlands, 1987. MR 89b:34001

[8] M. A. Naimark, Linear Differential Operators, Part II, Ungar, 1968. MR 41:7485

[9] F. Stenger, Approximations via Whittaker's cardinal functions, J. Approx. Theory, 17, 1976, 222-240. MR 58:1885

[10] E. C. Titchmarsh, Eigenfunctions expansion, Part II, Oxford University Press, 1961.

[11] A. I. Zayed, Advances in Shannon's Sampling Theory, CRC Press, 1993. MR 95f:94008

Department of Mathematics, College of Sciences, Sultan Qaboos University, P.O. Box 36, Alkhod 123, Muscat, Sultanate of Oman

E-mail address: boumenir@squ.edu.om 